\title{
ANÁLISIS DE ACCIDENTES SEVEROS EN CONTENCIONES NUCLEARES
}

\author{
(ANALYSIS OF SEVERE ACCIDENTS IN NUCLEAR CONTAINMENTS)
}

José M. a Goicolea Ruigómez, Dr. Ingeniero de Caminos E.T.S. de Ingeniero de Caminos de Madrid*.

Francisco Martínez Cutillas, Ingeniero de Caminos PRINCIPIA, S. A.

Felipe Gabaldón Castillo, Ingeniero de Caminos E.T.S. de Ingenieros de Caminos de Madrid*.

ESPAÑA

RESUMEN

El análisis frente a accidentes severos ha venido a añadirse recientemente a la larga lista de requisitos impuestos a las centrales nucleares. En este trabajo se pretende estudiar la problemática y justificación de este tipo de análisis. El cálculo de la respuesta de la contención se puede realizar en el ordenador mediante métodos avanzados de elementos finitos no lineales. Se hace una revisión del estado de la técnica en estos métodos, proponiendo una metodología basada en el análisis mediante modelos numéricos con validación experimental.

Se presentan asimismo los resultados de análisis realizados, para contenciones tanto de hormigón armado como

metálicas. Las conclusiones del trabajo establecen la validez de la metodología empleada, así como la constatación de un amplio margen de seguridad existente en las contenciones evaluadas, debido a los conservadurismos del proceso de diseño original.
SUMMARY

Analysis for severe accidents has recently been added to the long list of requisites imposed on nuclear power plants. In this work we study the rationale and justification for this type of analysis. Calculations for the structural response of the containment buildings can be performed on the computer using advanced nonlinear Finite Element codes. A state of the art review of these methods is carried out, proposing a methodology based on analysis by numerical methods with experimental validation.

Additionally we present some results obtained by the authors for the analysis of both reinforced concrete and steel containments. The concluding remarks show the validity of the proposed methodology, as well as the evidence of a large security margin in the containments, due mainly to the conservatisms in the design stage.

\section{INTRODUCCIÓN}

La industria nuclear ha generado durante las dos últimas décadas un importante impulso en diversas ramas de la ingeniería, y en particular en la tecnología de la construcción y la ingenieria civil. En este último campo, ha sido notorio el progreso en métodos de análisis dinámico para estructuras bajo solicitaciones sísmicas, o para análisis de recipientes a presión. EI desarrollo de los métodos de cálculo y diseño ha venido ligado también al progreso de los ordenadores digitales y a la implantación del cálculo por elementos finitos, lo que ha dotado a los ingenieros de medios de análisis sin precedentes.

\footnotetext{
* Anteriormente en PRINCIPIA, S. A
}

En este artículo presentamos algunos desarrollos recientes en esta línea, en particular para el análisis de contenciones nucleares frente a accidentes severos. El estudio de la seguridad de las instalaciones nucleares frente a este tipo de accidentes, no previstos en el diseño original de las plantas por su extremadamente baja probabilidad, ha venido motivado por la especial sensibilidad pública respecto a la energía nuclear.

En el resto de este trabajo describimos brevemente en primer lugar la problemática de los accidentes severos en centrales nucleares y sus metodologías de análisis. Seguidamente exponemos el estado de la técnica en análisis no lineal de estructuras. A continuación presentamos algunas aplicaciones, tanto para el análisis de contenciones de hormigón armado como para contenciones metálicas. Por último extraemos algunas conclusiones generales sobre la aplicabilidad de las técnicas descritas. 


\section{ACCIDENTES SEVEROS EN CENTRALES NUCLEARES}

\subsection{Accidentes severos y contenciones nucleares}

El edificio de contención es la última barrera ingenieril contra los vertidos incontrolados de productos de fisión al medio ambiente en caso de accidente. Hasta ahora las exigencias de diseño para las contenciones se han limitado a satisfacer la condición de retención en el supuesto de que no se produjese la fusión del núcleo. A raíz de los accidentes de Three Mile Island en 1978 y el más reciente de Chernobyl en 1986, los organismos reguladores y la industria nuclear han incrementado el interés en la determinación de las consecuencias de un accidente más allá de las condiciones de diseño.

Las bases tradicionales de diseño de contenciones han consistido en postular roturas en el circuito primario y secundario: DEGB's (Double-Ended Guillotine Breaks), LOCA's (Loss Of Coolant Accidents) y MSLB's (Main Steam Line Breaks). Estos sucesos se han identificado como los máximos liberadores de masa y energía y son usados tradicionalmente para calcular las máximas presiones y temperaturas en la contención. La metodología utilizada para diseñar frente a estos sucesos es extraordinariamente conservadora y garantiza un amplio margen de seguridad. Esto hace que en realidad puedan soportarse, no sólo las cargas de diseño, sino también muchas de las asociadas a accidentes de más baja probabilidad como los accidentes severos (29).

Se denomina Accidente Severo, según el Grupo de Expertos de la Comisión de Seguridad de Instalaciones Nucleares de la NEA, aquel que teniendo una probabilidad de ocurrencia muy baja y escapando por tanto a las condiciones de diseño de la Central, puede ocasionar un riesgo importante para el entorno (11). Un accidente severo puede estar originado tanto por causas externas -impacto de avión, terremoto superior al sismo de parada segura (SSE), explosiones externas,...como internas - cualquier suceso que ocasione la imposibilidad de refrigerar el núcleo-.

Las contenciones nucleares han adquirido un alto protagonismo como elemento "gestor" en situaciones de accidente (27). El conocimiento de su capacidad resistente real y por tanto del margen de seguridad nos permite en primer lugar conocer la seguridad de toda la planta en su conjunto, en segundo lugar gestionar el propio accidente tomando decisiones a seguir en función del nivel de presión y temperatura en el interior del edificio de contención y por último nos permite adecuar, con mayor conocimiento de causa, los distintos planes de emergencia (28).

\subsection{Metodología de análisis de accidentes severos}

El análisis de contenciones frente a accidentes severos tiene por objeto evaluar de manera realista (es decir, sin conservadurismos) los márgenes de seguridad existentes. En el caso en que se detectaran en las contenciones de las centrales existentes puntos débiles en los que el margen de seguridad fuese notoriamente inferior al resto de la contención, esta evaluación permite una mejora selectiva de dichos puntos incrementando asi de manera notable la seguridad de la central.

La determinación de la capacidad estructural última de la contención se puede hacer de dos maneras básicas:

1. Experimentación en modelos reducidos a escala de contenciones similares.

2. Análisis por ordenador mediante modelos numéricos adecuados.

La experimentación posee, además de su elevado coste, otro inconveniente importante frente a la simulación por ordenador. Se trata de la difícil - a veces incluso imposible- extrapolación de sus resultados a condiciones de carga o tipologías distintas a las ensayadas.

La simulación por ordenador constituye una herramienta más versátil, tanto por su coste más bajo como por su disponibilidad. Sin embargo, la exigencia a los modelos de cálculo es muy elevada, puesto que han de ser capaces de representar con suficiente fiabilidad y robustez comportamientos altamente no lineales, abarcando los estados últimos de fallo de la estructura y de los materiales.

Atendiendo a este tipo de necesidades, se ha desarrollado en los últimos años una actividad importante en investigación y desarrollo de métodos de elementos finitos no lineales. Estos métodos deben poder abarcar el comportamiento anelástico o incluso el fallo de los materiales, las grandes deformaciones y grandes desplazamientos, los fenómenos de contacto, el acoplamiento térmico-mecánico, etc.

A pesar de tratarse de un campo en desarrollo y en el que aún son necesarios avances importantes, se puede afirmar que hoy en día existen herramientas de cálculo disponibles, capaces de estudiar con éxito fenómenos complejos como la rotura por sobrepresión interna, impacto de avión o de misil, etc.

Dos tipos de dificultades se presentan al realizar estos análisis. Una, la derivada del desconocimiento de modelos teóricos adecuados para el comportamiento de los materiales. Otra, la necesidad de considerar en 
el análisis las distintas escalas existentes en estructuras grandes y complejas. Estas dificultades han de suplirse mediante la validación de los modelos con los resultados experimentales, proporcionando la necesaria confianza en el método.

Se han realizado últimamente varios programas para evaluar la integridad de contenciones, financiados por la Comisión de Regulación Nuclear (NRC) de los Estados Unidos y organismos de otros paises. En ellos se han efectuado ensayos de sobrepresión interna en diversos modelos reducidos de contenciones de acero, hormigón armado y hormigón pretensado. Uno de los objetivos principales perseguidos en estos programas ha sido comprobar la validez de los modelos de cálculo disponibles, para lo cual previamente a los ensayos se efectuaron los análisis pertinentes por parte de diversos organismos, siendo comparados después con los resultados experimentales realmente obtenidos.

Como datos básicos para el análisis se debe disponer en primer lugar de un "Postulado de Accidente", que se traduce generalmente en unas curvas de evolución de presión y temperatura con el tiempo, en el interior de la contención. En segundo lugar se necesitan las características geométricas concretas de la estructura así como las propiedades termomecánicas de los materiales, obtenidas mediante ensayos o de acuerdo a las especificaciones de diseño. Por último es necesario un criterio de fallo de la contención, generalmente relacionado con la pérdida de estanquidad de la misma, ya sea por fallo del material (fisuración excesiva en contenciones de hormigón, o por localización excesiva de deformaciones, en contenciones de acero) o por pérdida de estanquidad en los dispositivos de cierre.

Las herramientas de cálculo, basadas generalmente en el Método de los Elementos Finitos, han de ser capaces de reproducir comportamientos altamente no lineales con suficiente fiabilidad y robustez. Dichas no linealidades pueden ser de carácter geométrico: grandes movimientos de la estructura, contactos originados por dichos desplazamientos con estructuras colindantes, etc. También pueden venir ocasionadas por el comportamiento constitutivo de los materiales: plastificación, fisuración, dependencia de las propiedades mecánicas con la temperatura, etc.

Este tipo de análisis a pesar de la disponibilidad de ordenadores de gran potencia, requiere grandes tiempos de ejecución. Por esta razón resulta inviable la modelización de la estructura con todos sus detalles. El procedimiento generalmente aceptado es el realizar el análisis en sucesivas etapas obteniendo límites superiores de la capacidad resistente última (31). En una primera etapa es conveniente el analizar un modelo numérico que proporcione una respuesta "global" de la estructura sin representar efectos locales. Con este primer modelo podemos llegar a obtener una cota superior de la capacidad resistente asi como el modo global de colapso, de acuerdo al criterio de fallo establecido en las especificaciones.

En una segunda etapa, se realizarian modelos numéricos de "detalle" para el estudio de zonas singulares en la contención tales como penetraciones de conducciones, esclusas, juntas de cierre, etc. En esta etapa se utilizaría como condiciones de contorno del modelo las obtenidas del modelo global.

Por último, si fuera necesario para completar el estudio se pueden realizar ensayos reales para estimar el comportamiento de elementos difíciles de modelizar numéricamente.

\section{ANÁLISIS NO LINEAL DE ESTRUCTURAS}

El desarrollo de los métodos de análisis de estructuras en las últimas tres décadas ha venido muy ligado a los métodos numéricos de resolución por ordenador, y en particular al Método de los Elementos Finitos (MEF), que ha demostrado ser el método más potente y general de los conocidos.

No vamos a realizar aquí una descripción siquiera resumida del MEF, para lo cual se recomienda consultar las referencias $(1,2)$, sino más bien comentar los aspectos más relevantes del desarrollo del MEF especialmente en cuanto a aplicaciones no lineales se refiere.

La evolución del MEF se ha producido durante el último medio siglo, paralelamente al desarrollo de los ordenadores digitales. Después de que Courant (3) sentase las bases matemáticas del método en 1943, se atribuye a Argyris (4) la primera aplicación a elementos estructurales en 1954 y a Clough (5) de manera más general para elementos de continuo en 1960. La primera formulación completa del método para problemas no lineales, incluyendo grandes deformaciones y comportamiento elastoplástico, se publica en 1970 (6).

A pesar del trabajo realizado por la comunidad investigadora durante todos estos años, existen aún varias áreas de investigación en las que son necesarios avances importantes. Éstas incluyen:

- El análisis de fenómenos no lineales, incluyendo el desarrollo de modelos constitutivos para materiales estructurales en el rango no lineal.

- El desarrollo de estimadores de error y su incorporación en generadores de malla adaptativos. 
- La obtención de elementos más potentes y fiables, especialmente para placas y láminas.

- El análisis de problemas dinámicos, incluyendo choque e impacto a altas velocidades.

- Los problemas acoplados con efectos no estructurales, como térmicos, fluidos, etc.

En lo que sigue nos ceñiremos al primer aspecto citado, aunque es necesario mencionar que el desarrollo en esta línea viene condicionado por los avances en el resto de los puntos.

El comportamiento no lineal de una estructura puede provenir de diversas causas. En primer lugar hay que citar los efectos geométricos, producidos por las grandes deformaciones, rotaciones o desplazamientos. Estos efectos se expresan en la formulación del MEF mediante operadores no lineales en función de los desplazamientos, invalidando la aplicación de las medidas usuales propias de pequeñas deformaciones. Conviene destacar que aunque las deformaciones de las estructuras usuales de ingeniería son pequeñas - al menos en las condiciones de servicio- puede no ocurrir así con las rotaciones que a menudo son un orden de magnitud mayores, especialmente en estructuras esbeltas. Por otra parte, el análisis de solicitaciones últimas o accidentes sí requiere a menudo el análisis de grandes deformaciones. Existen fundamentalmente dos tipos de formulaciones para problemas de grandes deformaciones:

- Las formulaciones Lagrangiana (6) y Lagrangiana actualizada (7), que se basan en medidas totales de tensiones y deformaciones respecto a la configuración original, como el segundo tensor de Piola-Kirchhoff $S$ y el tensor de deformaciones de Green $\mathbf{E}$ (8). Estas formulaciones son preferibles para materiales de tipo hiperelástico en los que el comportamiento del material se refiere a un estado natural libre de cargas, como en los casos de cauchos, gomas, etc.

- Formulaciones incrementales, basadas en la configuración deformada (9), empleando el tensor de tensiones de Cauchy $\sigma$ y la velocidad de deformación d. Estas formulaciones tienen la ventaja de que se acoplan mejor a la teoria clásica de la plasticidad (10), que está expresada en función de la configuración deformada. Posee el inconveniente de que en un caso general las medidas incrementales de deformación no son integrables, al estar definidas respecto a configuraciones cambiantes.

Una exposición más detallada de estas formulaciones puede encontrarse en (12).
Otra causa importante de comportamiento no lineal es el material. El fenómeno más conocido es la plasticidad, por el cual se producen deformaciones remanentes en el material, al cargarlo por encima de su límite elástico. Existe una teoría clásica (10) relativamente bien establecida para explicar el comportamiento de materiales metálicos. Se han intentado generalizar los modelos elastoplásticos de comportamiento a otros materiales como los suelos o el hormigón, existiendo para ello multitud de modelos propuestos por distintos autores. Estos modelos se diferencian en el tipo de superficie propuesta, en los criterios de endurecimiento, o en la dirección de las deformaciones plásticas una vez que se alcanza la superficie de fluencia. Para los suelos podemos citar los modelos de Prévost (13) o Cam-Clay (14). En cuanto al hormigón, algunos de los modelos más generales son los de Willam (15), Ottosen (16) o Chen-Chen (17).

Una dificultad adicional para representar el comportamiento del hormigón es la marcada anisotropía de su respuesta, siendo muy distinta a compresión (para la que exhibe una resistencia y ductilidad considerables) y a tracción (resistencia del orden de 1/10 de la de compresión y rotura frágil por fisuración). Existen dos procedimientos que se han empleado en la práctica en el MEF para tratar la fisuración. El modelo de fisura distribuida considera la fisuración como un daño distribuido en una zona del material de ancho finito determinado. El otro procedimiento es el de la fisura discreta, para el que la fisura es una grieta que produce una discontinuidad geométrica y topológica en el modelo, separándose los labios de la misma. Los procedimientos de fisura discreta permiten incorporar directamente criterios de mecánica de la fractura, aunque no son aplicables con generalidad a casos en que se produzcan múltiples fisuras en direcciones y puntos no previstos de antemano. Su utilización actualmente se restringe para el estudio detallado de grietas individuales en lugares prefijados. Los modelos de fisura distribuida se acoplan mejor al cálculo por elementos finitos para casos generales. Sin embargo es necesario evitar problemas fundamentales como la dependencia de la solución del tamaño de la malla (18).

Otros efectos no lineales debidos al material son la variación de sus propiedades con la temperatura, la rotura o reblandecimiento, el daño o degradación de sus constantes elásticas, y fenómenos viscoplásticos o de fluencia.

Conviene recalcar que uno de los campos en que más se necesita avanzar es en los modelos constitutivos de materiales. En este terreno el progreso es más lento, al ir necesariamente ligado a investigaciones experimentales complejas. Desconocemos aún en gran medida cómo se comportan muchos materiales que empleamos en ingeniería, con el suficiente detalle co- 
mo para formular modelos de comportamiento detallado que alimenten a los modelos de elementos finitos disponibles. Esto es así ciertamente para muchos de los nuevos materiales (cerámicos, compuestos, etc.) pero también para algunos materiales considerados tradicionales como el hormigón.

Por último, existe otra fuente importante de efectos no lineales, aquéllos producidos por efectos de borde. Éstos incluyen los contactos o despegues en el contorno, el rozamiento por deslizamiento en el mismo, el corrimiento de fuerzas o sobrecargas, etc.

Para abarcar estas fuentes de comportamiento no lineal se necesitan tipos de elementos especialmente adecuados, ya que existen diversos problemas que no aparecen en los modelos lineales. Un problema bien conocido es el bloqueo de la malla para flujo plástico incompresible, circunstancia que se presenta en ciertos tipos de mallas (19). Esto ocurre por ejemplo en los triángulos y tetraedros de 1 punto de integración (deformación constante), y en los cuadriláteros con integración completa $2 \times 2$. Una solución para este problema es la subintegración de las componentes volumétricas, dando lugar a las técnicas de integración reducida (1) o la $\bar{B}$ de Hughes (2).

En esquema, se debe buscar elementos:

- simples y eficaces,

- libres de bloqueo de la malla,

- de rango adecuado (es decir, sin modos de energía nula),

- insensibles a la distorsión,

- que proporcionen respuestas precisas aun con mallas gruesas,

- aplicables para problemas no lineales y dinámicos.

Quizá los elementos que ofrecen mayores dificultades para cumplir estos requisitos son los elementos de placa o lámina, tanto gruesa como delgada (20). Las tendencias de desarrollo reciente son las de utilizar formulaciones mixtas - con los desplazamientos y el cortante como variables - (21) o campos de deformaciones impuestas (22).

Un modelo no lineal que combine varias fuentes de nolinealidad como las mencionadas anteriormente, una vez discretizado mediante elementos finitos, da lugar a una ecuación no lineal en desplazamientos del tipo.

$$
\mathrm{f}(\mathrm{u})=\mathrm{F},
$$

donde $\mathbf{u}$ son los desplazamientos nodales incógnita, $f(u)$ las fuerzas internas del material, y $F$ las acciones exteriores. En los casos lineales se verifica $f(u)=K \cdot u$, y la ecuación anterior se resuelve simplemente mediante la inversión de la matriz de rigidez K. Por el contrario en el caso no lineal es necesario emplear métodos de solución iterativos hasta alcanzar la convergencia. El método más conocido es el de Newton, que emplea la matriz de rigidez tangente $K_{t}=\frac{d f}{d \mathbf{u}}$ para las su-
cesivas iteraciones.

$$
u^{i+1}=u^{i}-\frac{\left(f^{i}-F^{i}\right)}{K_{t}^{i}}
$$

El método de Newton se puede emplear en su versión modificada, en la que se evita reformular $\mathrm{K}_{t}$ para cada ciclo de la iteración, lo que es adecuado para problemas débilmente no lineales. Otras alternativas son los métodos cuasi-Newton (23), el gradiente conjugado o la relajación dinámica (24). Para situaciones en que $\mathrm{K}_{t}$ se hace singular, es necesario emplear técnicas como la de la longitud de arco (25) para su resolución.

La resolución de problemas no lineales exige por último el empleo de programas de ordenador avanzados, cubriendo la problemática citada anteriormente. Así, aunque muchos programas de Elementos Finitos ofertados en el mercado anuncian la capacidad de resolver problemas no lineales, en realidad la mayoría de ellos tienen unas prestaciones muy limitadas en este campo, o bien su fiabilidad deja mucho que desear. Entre los programas que han sido empleados con éxito para análisis no lineales de estructuras de contención cabe citar a ABAQUS (35) y ADINA (26).

Por otra parte, los programas no lineales exigen recursos de ordenador cuantiosos, por lo que son difícilmente utilizables en ordenadores personales para estructuras reales de ingeniería. Por este motivo, la mayoría de los programas no lineales necesitan ordenadores al menos del tipo estación de trabajo ("Workstation") u ordenadores grandes multi-usuario. Aunque con el ritmo de desarrollo en prestaciones de los ordenadores, es probable que dentro de poco estos problemas sean también abordables desde PC's.

\section{APLICACIÓN A LAS CONTENCIONES DE HORMIGÓN ARMADO}

\subsection{Dificultades en la modelización del hormigón}

La modelización numérica de contenciones de hormigón armado y pretensado resulta ser algo más compleja que las correspondientes de acero. Esta dificultad radica en la complejidad de representar el modelo constitutivo del hormigón.

El hormigón es un material de gran difusión en la industria de la construcción. Existe una gran unanimi- 
dad en las metodologías de análisis orientadas al diseño de estructuras de hormigón, lo que se traduce en códigos y normativas de diseño que poseen un carácter supranacional. Estas metodologías a pesar de haber demostrado su eficiencia en la comprobación de la seguridad, no resultan adecuadas para la obtención de respuestas realistas de contenciones como en el caso que nos ocupa. La razón estriba en que resulta necesario el conocimiento de las distintas etapas de fisuración y la evolución del nivel de deformaciones con el nivel de presión; en definitiva un conocimiento más exhaustivo paso a paso de la respuesta estructural de la contención. En este sentido no existen criterios unificados en el modo de abordar el problema, dejándose muchas veces en manos del analista numérico la adopción de hipótesis, modelos de material, etc, que pueden llegar a ser condicionantes del propio resultado.

Con el objetivo de efectuar una revisión de la capacidad de análisis en este terreno, se ha realizado recientemente un estudio (32) en el que se analizan los modelos constitutivos existentes para representar el comportamiento del hormigón, susceptibles de emplearse en el cálculo de una contención frente a accidentes severos. Asimismo se estudia el rango de aplicabilidad que deben cumplir los modelos de cálculo y las técnicas numéricas de resolución.
La dificultad de modelización numérica del hormigón radica en la desigual capacidad resistente en tracción frente a compresión. La baja resistencia a tracción hace que a tensiones relativamente bajas el hormigón comience a fisurarse, originando una pérdida de rigidez progresiva al mismo tiempo que se produce una transferencia de carga a las armaduras. Este proceso resulta de por sí numéricamente inestable. Juega un papel muy imporante en esta fase la propia robustez de los códigos numéricos, así como el control de los parámetros de convergencia. Una vez superada esta fase, el comportamiento viene gobernado básicamente por el acero de las armaduras.

\subsection{Modelización de la contención de hormigón Sandia Escala 1:6}

\subsubsection{Antecedentes}

Describiremos algunos aspectos más relevantes de la simulación realizada (33) para reproducir uno de los experimentos del Laboratorio Nacional Sandia dentro del Programa de Integridad de Contenciones (34). Dicho modelo corresponde a una contención típica de un reactor de agua a presión (PWR) de hormigón armado, a escala 1:6.



Fig. 1.-Modelo a escala 1:6 de una contención de hormigón armado sometida a presurización interna en el Laboratorio Nacional Sandia (EEUU). 
El modelo a escala de la contención (figura 1), es una lámina cilíndrica de $3,353 \mathrm{~m}$ de radio cubierta con una cúpula semiesférica. El espesor oscila entre $17,8 \mathrm{~cm}$ y $24,8 \mathrm{~cm}$ y se encuentra interiormente revestida por un "liner" de acero de 1,60 mm. Este modelo fue sometido a un incremento gradual de la presión mediante gas nitrógeno. Al aumentar la presión se produjo un desgarro y el experimento concluyó cuando las bombas no podían suministrar el suficiente caudal para compensar las fugas existentes. Las fugas se localizaron primordialmente a través de una fisura en el "liner" de acero cercano a una penetración de la contención (figura 2), cuando la presión era aproximadamente de $1 \mathrm{MPa}$.

Los análisis numéricos previos fueron bastante coincidentes en la detección del modo de colapso global a partir de la presión de 1,1 $\mathrm{MPa}$, sin embargo no fueron capaces de prever el modo de fallo localizado.

\subsubsection{Objetivos de la simulación}

Los objetivos de la modelización a nivel genérico han sido, la validación de una metodología de cálculo así como el establecimiento de herramientas necesarias para el cálculo de contenciones reales de hormigón. De una manera más específica, el objetivo es el cálculo de la capacidad última y modo de fallo de una contención de hormigón armado.

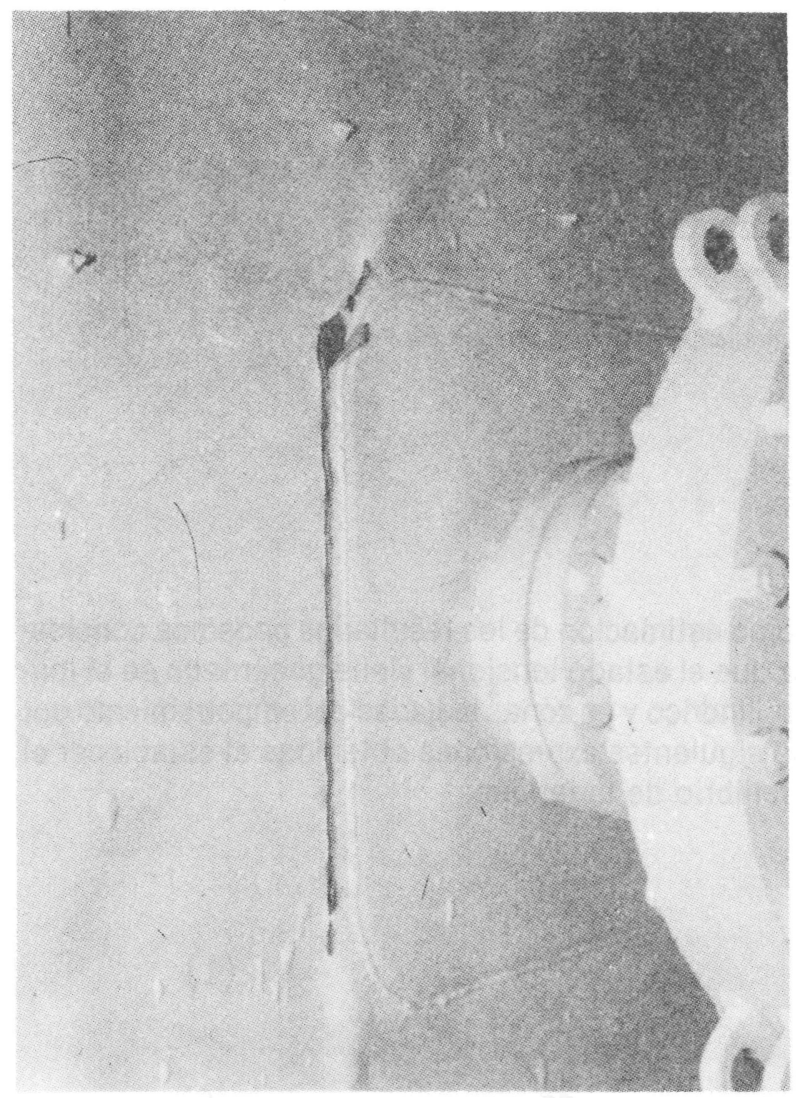

Fig. 2.-Zona del rasgado del "liner" donde se produjo el fallo es. tructural del modelo a escala 1:6.

(C) Consejo Superior de Investigaciones Científicas

Licencia Creative Commons 3.0 España (by-nc)

\subsubsection{Metodología de modelización}

Las características geométricas de la modelización objeto de estudio quedan recogidas en la figura 3. De acuerdo a dicha figura, la geometría es prácticamente axisimétrica, salvo en las penetraciones que rompen dicha simetría.

Debido a esta característica, se ha optado en una primera fase por establecer una modelización bidimensional axisimétrica sin tener en cuenta las penetraciones (figura 4). En una segunda fase se realizó un modelo tridimensional (figura 5) con objeto de evaluar las concentraciones de deformación y posibles modos de fallo local, aunque en los siguientes apartados nos centraremos en el modelo bidimensional. Dicha modelización se ha efectuado con el programa de Elementos Finitos de propósito general ABAQUS (35). Se ha tratado de reproducir lo más fielmente la geometría de la contención: losa de cimentación, pared cilíndrica, cúpula semiesférica, "liner" de acero, así como la disposición exacta de las armaduras.

El hormigón se ha modelizado mediante elementos axisimétricos lineales de cuatro nodos. Las armaduras se han representado mediante elementos "rebar", elementos unidimensionales que se superponen a los elementos sólidos de hormigón y se reparten uniformemente a lo largo del espesor de los mismos. El "liner" de acero se ha representado mediante elementos lámina axisimétricos de interpolación lineal de dos nodos.

La modelización de los materiales se ha realizado buscando la respuesta más allá del límite elástico y/o de fisuración, de la manera más realista posible. Así, el hormigón se ha representado como un material elastoplástico con endurecimiento en compresión, con superficie de carga según el modelo de Chen-Chen modificado incorporado en ABAQUS (35). En tracción se emplea un modelo de fisuración distribuida con ablandamiento. El comportamiento del acero tanto del "Iiner" como de las armaduras se ha representado como material elastoplástico isótropo con endurecimiento, con superficie de fluencia y rotura de Von Mises.

\subsubsection{Metodología de análisis}

La dificultad del análisis radica no tanto en la complejidad del estado tensional debido a la geometría estructural, como en las características de los materiales empleados. El comportamiento constitutivo del hormigón se caracteriza por una asimetría de funcionamiento en tracción y compresión. Éste es uno de los factores básicos diferenciadores de otros materiales resistentes de uso ingenieril.

El estado tensional de la contención objeto de estudio es fundamentalmente de tracción. Debido a que la resistencia a tracción es baja, del orden del diez por ciento de la de compresión (en el caso que nos ocupa http://informesdelaconstruccion.revistas.csic.es 




Fig. 3.-Características geométricas de la contención de hormigón a escala 1:6.

en torno a $4 \mathrm{MPa}$ ), las tensiones a las que va a estar sometida la estructura superarán con creces dicho vaIor. Esta pérdida de capacidad resistente se traduce en una fisuración del hormigón y en una transferencia tensional a las armaduras. Este proceso es numéricamente inestable. Ello hace que la convergencia numérica del modelo sea muy sensible a variaciones de parámetros tales como: resistencia a tracción del hormigón, pendiente de la rama de ablandamiento, etc. La dificultad en la convergencia de los análisis numéricos se ve agudizada por la propia distribución tensional de estas estructuras (láminas cilíndricas con cúpula semiesférica) cuando se encuentran sometidas a presurización interna.
Como estimación de los resultados podemos considerar que el estado tensional viene gobernado en el muro cilíndrico y en zonas alejadas del empotramiento por las siguientes expresiones obtenidas al establecer el equilibrio de la lámina: 




donde $\sigma_{z}$ es la Tensión Vertical o Meridional

$$
\begin{array}{ll}
\sigma_{\theta} & \text { Tensión Circunferencial } \\
r & \text { Radio de la Lámina Cilíndrica } \\
t & \text { Espesor de la Lámina Cilíndrica }
\end{array}
$$

Análogamente el estado tensional en el casquete semiesférico se puede expresar mediante:

$$
\begin{aligned}
& \sigma_{\phi}=\frac{p r}{2 t} \\
& \sigma_{\theta}=\frac{p r}{2 t}
\end{aligned}
$$

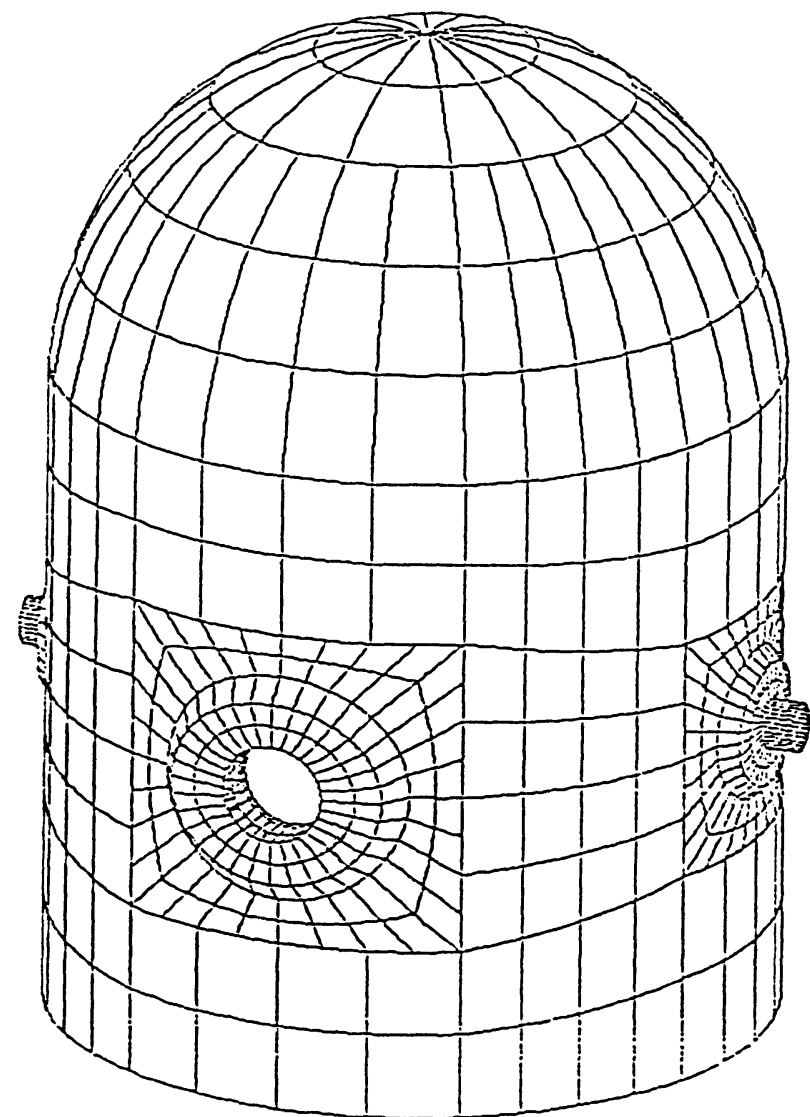

Fig. 5.-Discretización tridimensional de la contención de hormigón a escala 1:6.

Fig. 4.-Discretización bidimensional de

la contención de hormigón a escala 1:6.

\section{donde $\sigma_{\phi}$ es la Tensión Meridional \\ $\sigma_{\theta} \quad$ Tensión Circunferencial}

Por tanto la distribución tensional es la de tracción biaxil. De acuerdo a las expresiones anteriores y como más adelante verificaremos, la fisuración se inicia en el muro cilíndrico en el sentido circunferencial (debido a las tensiones circunferenciales), extendiéndose posteriormente en el sentido meridional (debido a las tensiones meridionales), generalizándose a lo largo de la cúpula semiesférica.

Cuando se produce la fisuración generalizada, el análisis no puede continuar debido a la falta de conver- 
gencia. No fue posible por tanto el realizar un análisis completo con un solo modelo que representase tanto las etapas previas de fisuración como las del estado último. Ha sido preciso realizar dos tipos de análisis bien diferenciados:

- Análisis de baja presión. Se corresponde con la aplicación de una presión interior desde 0 a 0,9 MPa. En dicho intervalo es donde se produce la completa fisuración del hormigón. Esta etapa viene gobernada por el comportamiento en tracción del hormigón y el proceso de transferencia tensional de éste a las armaduras. Se ha utilizado un modelo de hormigón con fisuración distribuida y ablandamiento. Se ha realizado un estudio de sensibilidad en el que se ha variado la pendiente de ablandamiento posterior a la fisuración. El caso I corresponde a una rama de ablandamiento casi vertical con deformación última de 0,001 , mientras el caso II corresponde a una deformación última de 0,005.
- Análisis de alta presión. Se corresponde con la aplicación de una presión interior desde 0 a 1,2 $\mathrm{MPa}$. A altas presiones, esto es a 0,9 MPa, el hormigón se encuentra totalmente fisurado, por tanto el comportamiento estructural viene gobernado por el acero de las armaduras y del "liner". El hormigón se ha considerado como un material elastoplástico con una resistencia a tracción despreciable.

\subsubsection{Análisis de los Resultados}

En el análisis de baja presión se detectaron tres etapas básicas de fisuración. Dichas etapas correspondieron a la fisuración circunferencial en el muro cilíndrico $(0,32 \mathrm{MPa})$, a la fisuración meridional del mismo así como a la fisuración circunferencial de la cúpula $(0,59 \mathrm{MPa})$ y por último cuando toda la contención se encuentra fisurada $(0,94 \mathrm{MPa})$.

Para la comparación de resultados se ha seleccionado la altura media del cilindro como punto de referen-

\section{EXPERIMENTO SANDIA ESCALA 1:6 Comparación de Resultados (Baja Presión)}
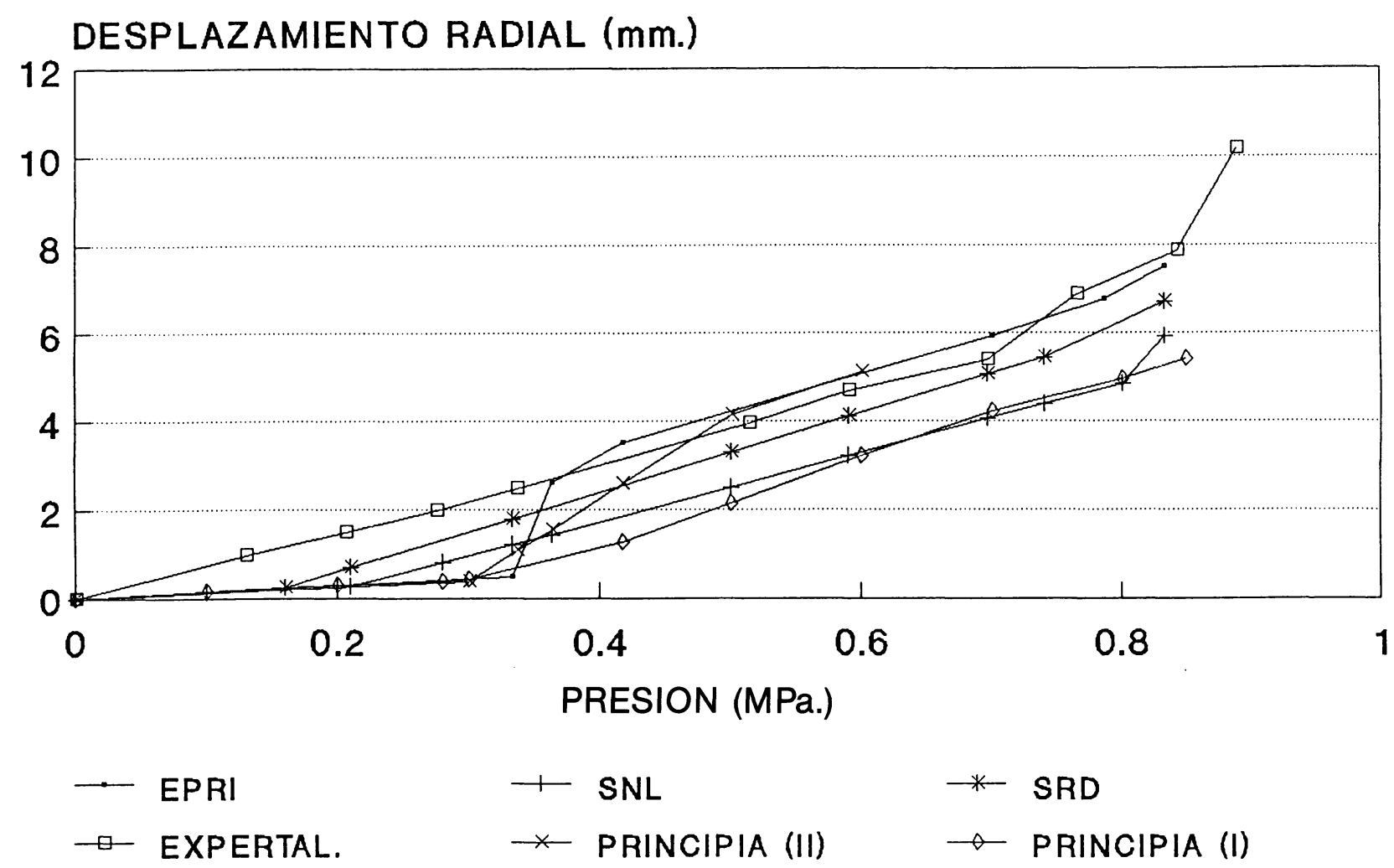

\section{ALTURA MEDIA DEL CILINDRO}

Fig. 6. - Comparación de resultados del desplazamiento radial a la altura media del cilindro del modelo de baja presión de la contención de hormigón a escala 1:6. 
cia. Se han comparado los resultados obtenidos con los experimentales así como los obtenidos por otras organizaciones que han realizado la simulación numérica: Electric Power Research Institute (EEUU), Sandia National Laboratory (EEUU) y Safety and Reliability Directorate (Reino Unido).

La figura 6 muestra la comparación de resultados de los desplazamientos radiales en los supuestos I y II. En dicha figura se puede apreciar fácilmente las dos rigideces de la estructura (doble pendiente de la curva) que se corresponden con la estructura fisurada y sin fisurar respectivamente. Existe un buen acuerdo en los valores de la rigidez fisurada, sin embargo todos los análisis tienden a sobrevalorar la rigidez previa a la fisuración con respecto a los medidos experimentalmente. Por otro lado se observa la influencia de la pendiente de la rama de ablandamiento, cuanto menor es ésta, mayor es la rigidez del hormigón fisurado.
La figura 7 recoge la comparación de resultados en el mismo punto de los desplazamientos verticales.

Para poder analizar los resultados más allá de los 0,9 $\mathrm{MPa}$ de presión interior, es preciso hacer referencia a los resultados obtenidos de alta presión. La figura 8 nos muestra la comparación de desplazamientos radiales a la altura media del cilindro, destacando su buen ajuste. La figura 9 muestra la distribución de deformaciones plásticas efectivas, cuando la presión alcanza el valor de 1,1 MPa, alcanzando valores máximos del $2 \%$. La figura 10 representa la evolución de la deformación de las armaduras circunferenciales a la altura media del cilindro.

A partir de la presión interna de 1,1 $\mathrm{MPa}$ se observa que las deformaciones plásticas tienen un crecimiento muy rápido. Se puede considerar este valor como presión última de fallo global, lo que equivale a tres veces la presión de diseño.

\section{EXPERIMENTO SANDIA ESCALA 1:6 Comparación de Resultados (Baja Presión)}

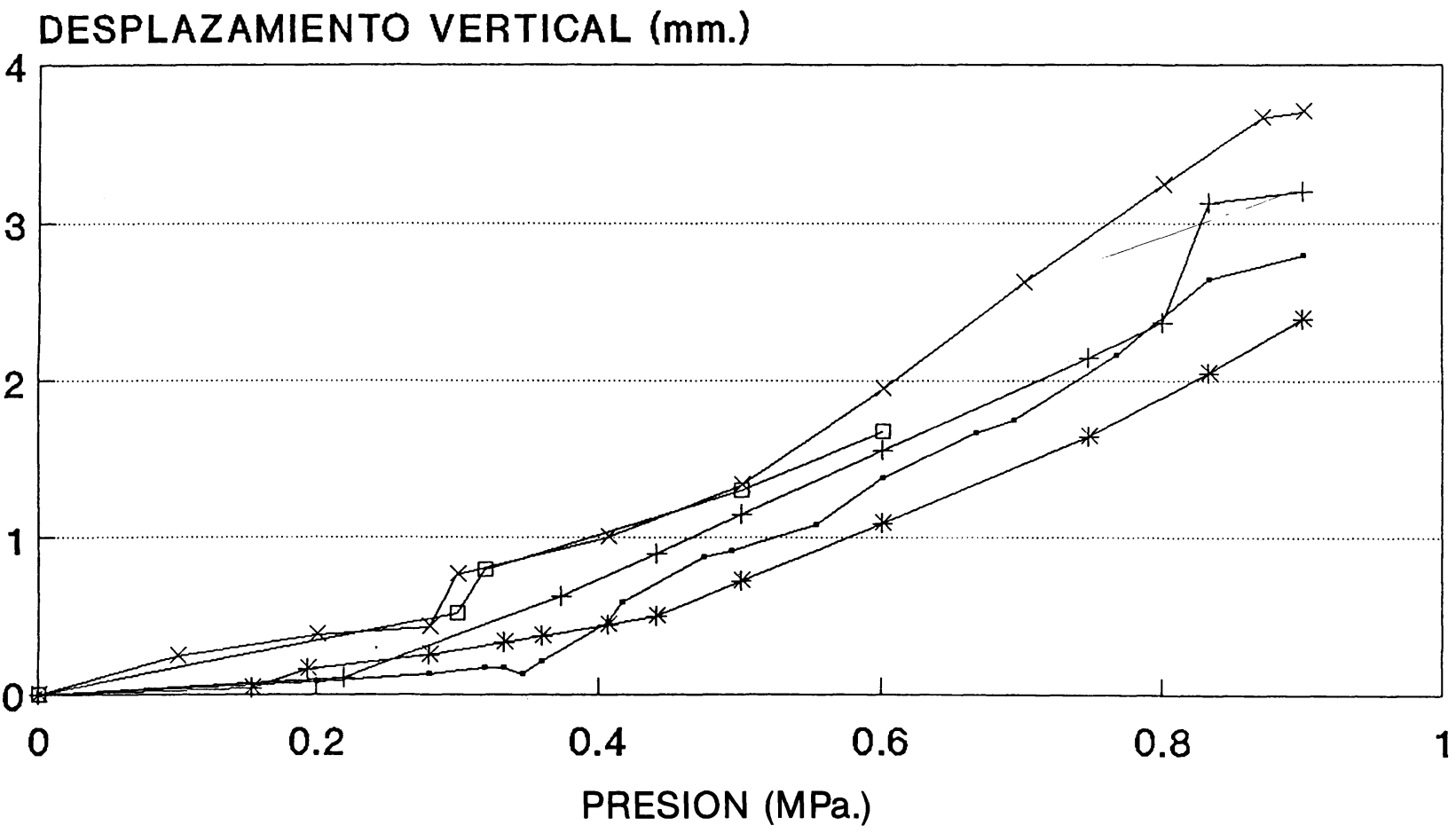

- EPRI + SNL * SRD —PRINCIPIA (II) $\rightarrow$ PRINCIPIA (I)

\section{ALTURA MEDIA DEL CILINDRO}

Fig. 7.-Comparación de resultados del desplazamiento vertical a la altura media del cilindro del modelo de baja presión de la contención de hormigón a escala 1:6. 


\section{EXPERIMENTO SANDIA ESCALA 1:6 Comparación de Resultados (Alta Presión)}

DESPLAZAMIENTO RADIAL (mm.)

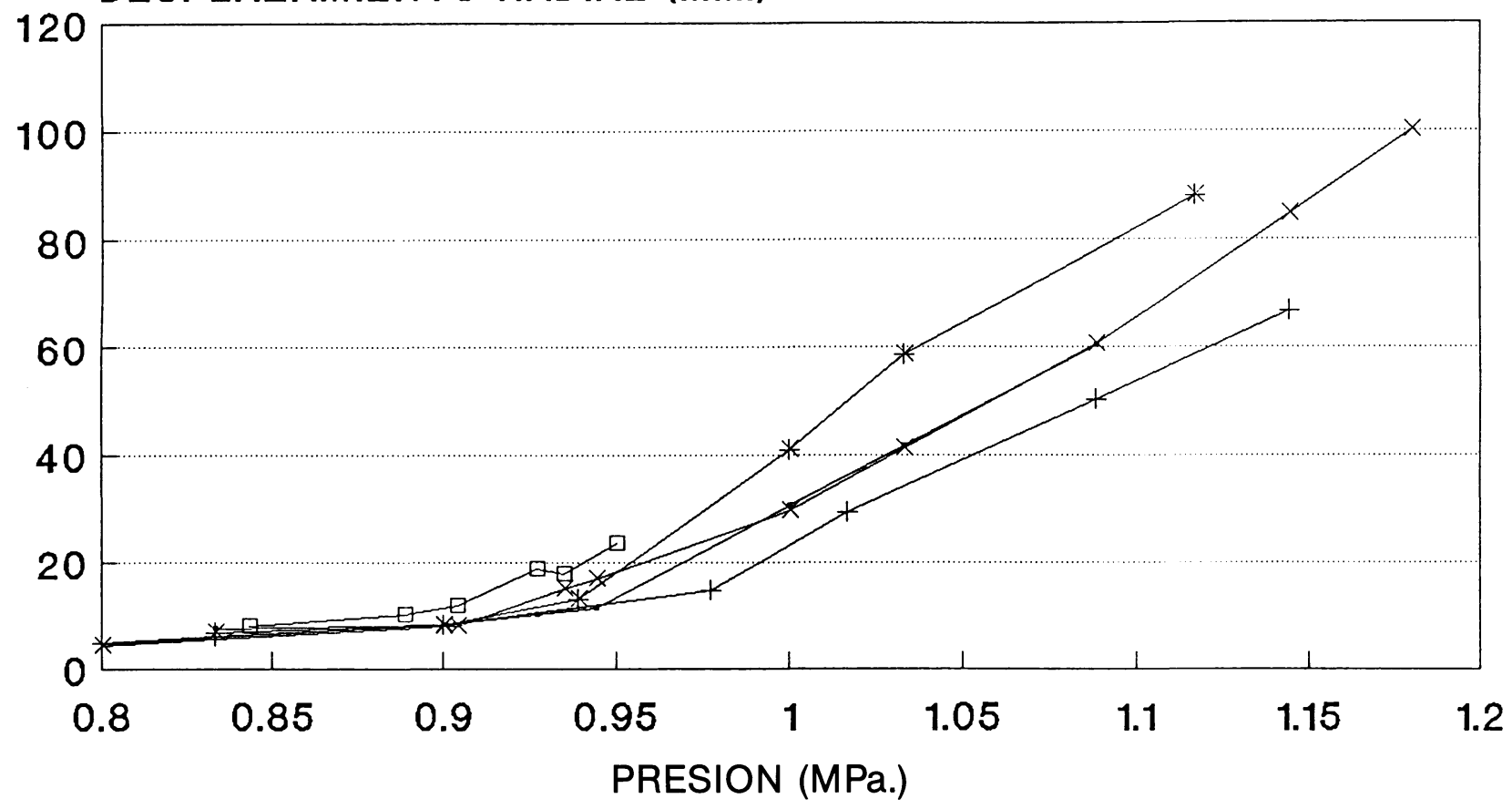

- EPRI $\rightarrow$ SNL $\quad *$ SRD $\quad$ - EXPERTAL. $\quad *$ PRINCIPIA

ALTURA MEDIA DEL CILINDRO

Fig. 8.-Comparación de resultados del desplazamiento radial a la altura media del cilindro del modelo de alta presión de la contención de hormigón a escala 1:6.

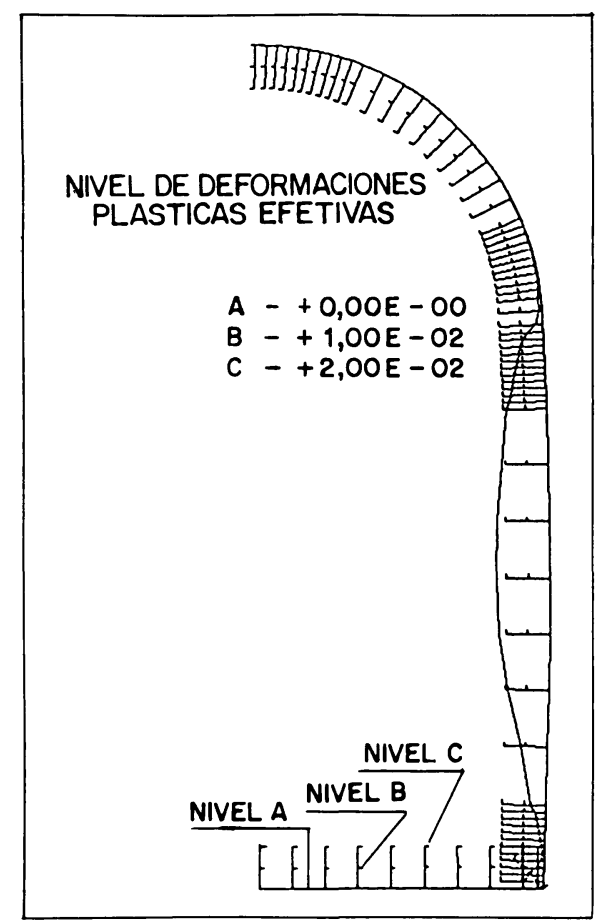

Fig. 9.-Nivel de deformaciones plásticas efectivas en el liner de acero a la presión de 1,1 MPa de la contención de hormigón a escala 1:6.

\section{ANÁLISIS DE CONTENCIONES METÁLICAS}

\subsection{Análisis efectuados}

Presentamos aquí algunos resultados de la evaluación de una contención metálica correspondiente a un reactor de agua en ebullición General Electric tipo Mk I.

La Contención Mk I (figura 11) está constituida por un cuerpo central en forma de bombilla invertida (Drywell), rodeado por un toro en su parte inferior (Wetwell), que se encuentra inundado de agua y conectado a la bombilla central a través de unos tubos de venteo. La bombilla central y el toro constituyen conjuntamente el recinto de contención sometido a sobrepresión interna en caso de accidente. El comportamiento desacoplado de ambas partes hace que sea posible estudiarlas independientemente, centrándose los modelos de elementos finitos que se describen en este apartado en la modelización del Drywell. La bombilla central consta de tres partes: una cúpula superior con forma de casquete esférico que constituye el cierre superior de la Contención, atornillado a una parte cilíndrica intermedia, y por último una parte esférica inferior que se empotra en la cimentación del reactor, unida a la lámina cilíndrica mediante acuerdos circulares. 


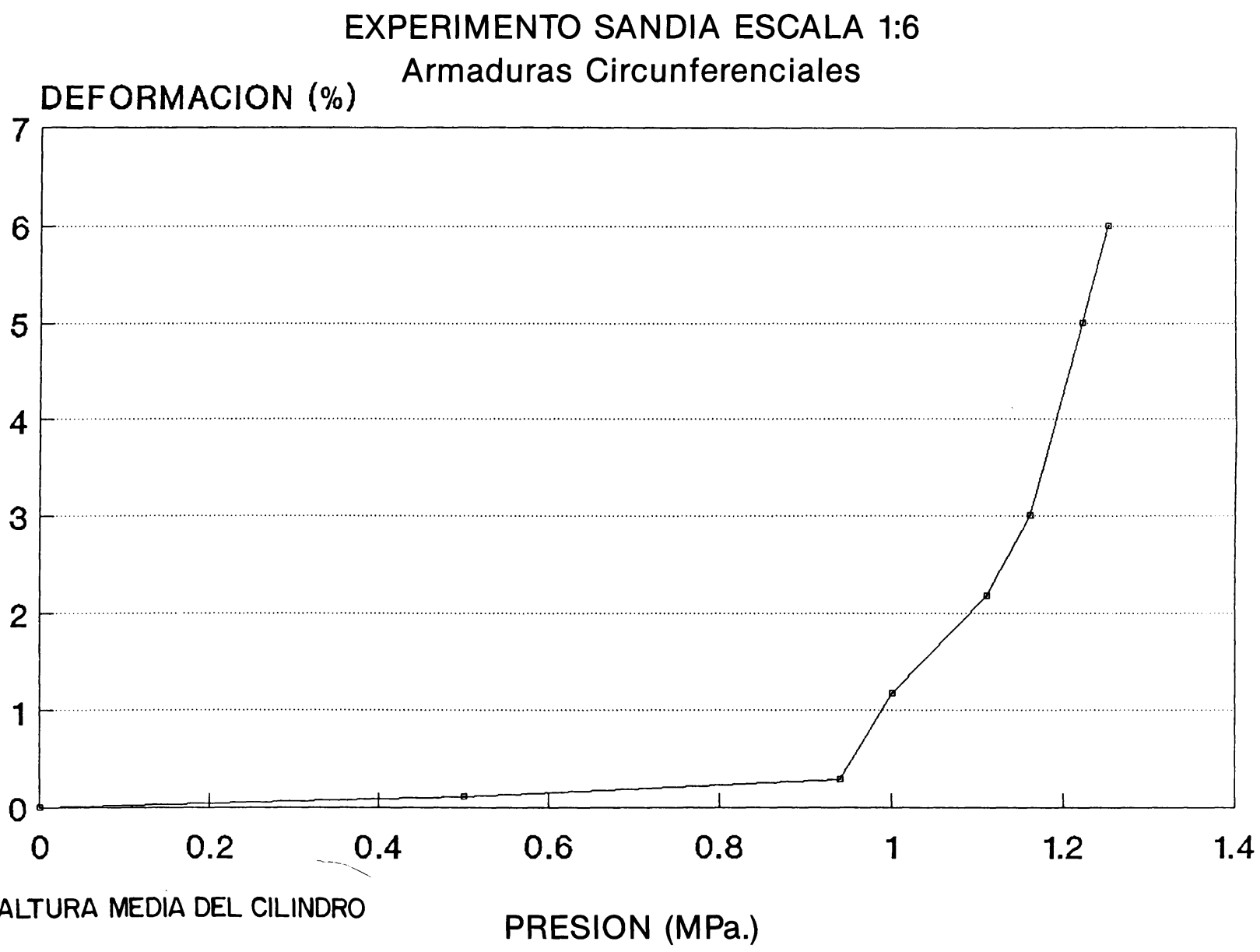

Fig. 10.-Evolución de las deformaciones de las armaduras circunferenciales a la altura media del cilindro del modelo de alta presión de la contención de hormigón a escala 1:6.

Dentro de la bombilla central se encuentra el muro de sacrificio, dispuesto entre el reactor y la contención y cuya función primordial es actuar como barrera biológica en caso de accidente.

\subsection{Metodología empleada}

El procedimiento empleado para los análisis consistió en realizar primeramente un modelo global de la Estructura de Contención (figura 11) que permitió conocer la presión y temperatura que produciría el colapso global de la estructura y el correspondiente modo de faIlo. Asimismo se obtuvieron las historias de movimientos y esfuerzos en la estructura a lo largo del tiempo que duraba el accidente. A continuación se realizaron varios modelos de detalle: penetraciones típicas (figura 12), puerta de equipos (figura 13), etc. para determinar los modos de fallo local.

El análisis de la estructura se debe realizar con un modelo tridimensional, representando correctamente el comportamiento no lineal de la misma, debido a la existencia de grandes deformaciones, la presencia de fenómenos de contacto en los contornos con el respaldo de hormigón que envuelve a la lámina metálica y el comportamiento elastoplástico del material.

No considerar estas características adoptando un exceso de hipótesis simplificadoras conduciría a la obtención de un comportamiento conservador pero poco realista de la estructura. Esto iría directamente en contra del objetivo de los análisis de accidente severo, en que se busca evaluar de manera realista la respuesta.

Los tipos de elementos finitos empleados en los modelos incluyen elementos lámina de ocho nodos, elementos viga de tres nodos, elementos de tipo sólido rígido, elementos muelle de dos nodos, etc.

El material para la lámina de la Contención y los tubos de venteo (acero) corresponde a un modelo elastoplástico de Von Mises, con propiedades que varían en función de la temperatura. El muro de sacrificio se supone que sólo resiste en estado de compresión triaxil debido a las fuertes oscilaciones térmicas que ha sufrido; sus características mecánicas se han definido por el módulo de elasticidad $E$, el coeficiente de Poisson $\nu$ y el coeficiente de dilatación lineal $\alpha$, parámetros dependientes de la temperatura. 

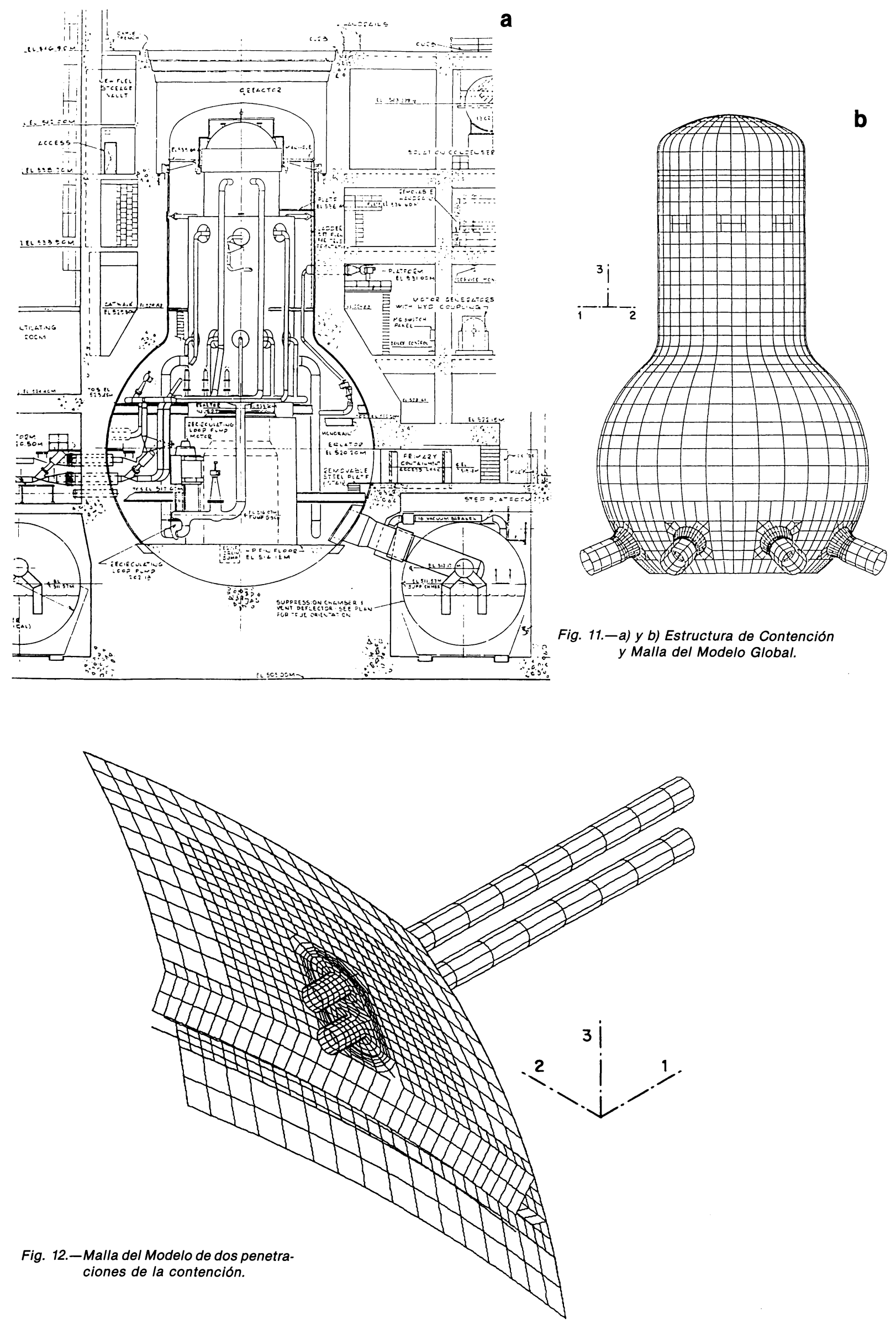


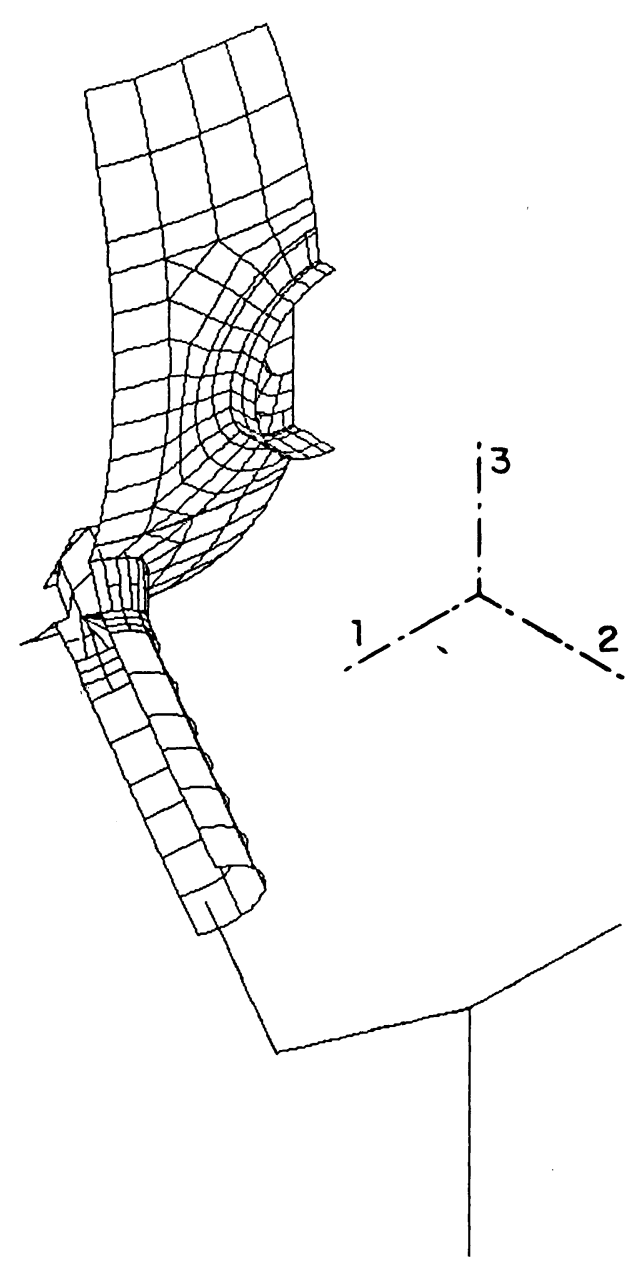

Fig. 13.-Malla del Modelo de la puerta de equipos.

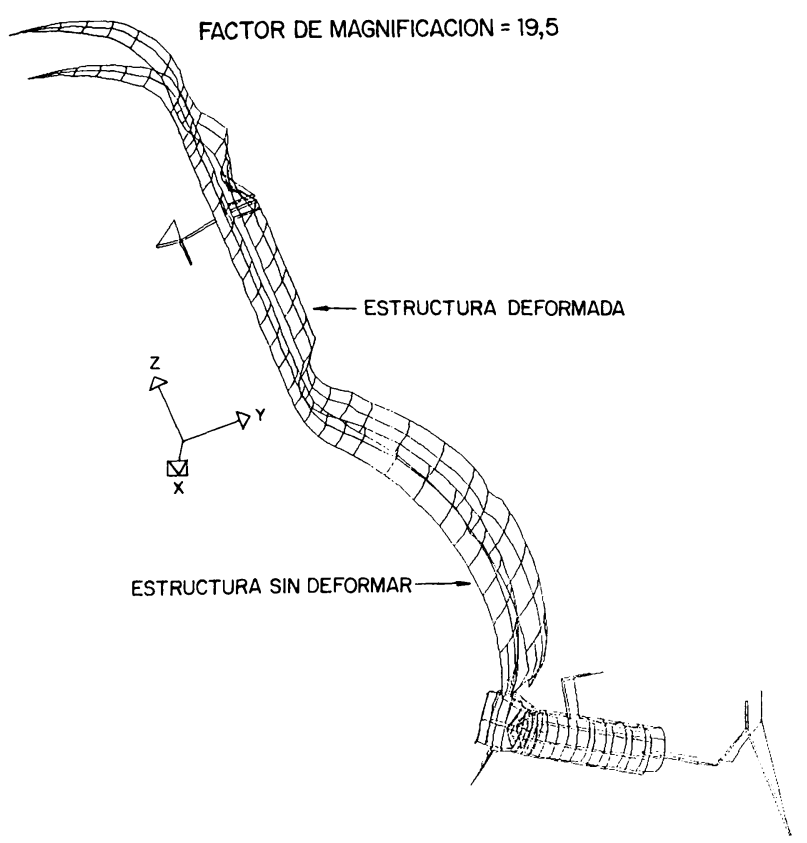

Fig. 14.-Resultados análisis global. Estructura deformada. (C) Consejo Superior de Investigaciones Científicas Licencia Creative Commons 3.0 España (by-nc)
El modelo se sometió a cargas consistentes en historias temporales predefinidas de presión interna en el interior de la lámina metálica de la Contención y en las líneas de venteo, e incremento uniforme de la temperatura en la Estructura de Contención, sin gradientes térmicos en el espesor de la lámina. Estas cargas se definieron de acuerdo con un escenario de accidente severo ATWS (Anticipated Transient Without Scram). La variación de las cargas es lo suficientemente lenta como para despreciar los efectos dinámicos. En los análisis de detalle además de la presión interna y de la temperatura se han impuesto en los contornos los desplazamientos y esfuerzos resultantes del análisis global, también en forma de historia temporal.

\subsection{Criterios de fallo}

El acero que constituye la lámina de la Contención es un material dúctil, sobre todo a las temperaturas que se postulan para el accidente, por lo que no es de esperar que se produzcan roturas de tipo frágil sino el fallo por desgarramiento, cuando la deformación plástica efectiva $\left(\epsilon_{\text {eff }}\right)$ alcanza un valor entre el $10 \%$ y el $15 \%$.

Los valores adoptados como criterio de rotura son los correspondientes a $\epsilon_{\text {eff }}=1 \%$ para estados tensionales de membrana, $\epsilon_{\text {eff }}=2 \%$ para estados tensionales de flexión y $\epsilon_{\text {eff }}=5 \%$ para las zonas muy localizadas. Estos valores son conservadores pero proporcionan una estimación razonable para determinar la capacidad última de la Contención ya que una vez alcanzados, se produce una localización de las deformaciones que conduce a un crecimiento muy rápido del daño.

\subsection{Resultados}

Los resultados obtenidos (figura 14) indican que la lámina se comporta globalmente en estado membrana salvo en las zonas próximas a ciertos puntos singulares, en las que predominan los estados de flexión. En estas zonas es donde se produce el colapso de la estructura con carácter local. La rotura se produce de forma dúctil observándose que a partir del instante en que aparecen las primeras deformaciones plásticas, éstas aumentan muy rápidamente aunque los incrementos de presión sean pequeños.

El contacto de la lámina metálica con el respaldo de hormigón ocasiona unas coacciones en los desplazamientos que es importante representar para obtener una modelización realista. A partir del contacto la respuesta de la estructura no cambia sustancialmente salvo en las zonas sin respaldo de hormigón o en aquellas en que se produce una variación de la separación lámina-respaldo.

http://informesdelaconstruccion.revistas.csic.es 


\section{CONCLUSIONES FINALES}

El análisis estructural frente a solicitaciones extremas, como es el caso de la evaluación de contenciones nucleares frente a accidentes severos, exige una modelización realista (no conservadora), para lo cual generalmente no resultan válidas las normas e hipótesis asumidas en la fase de diseño. Los dos procedimientos posibles de evaluación -experimentación en modelos a escala y análisis numérico por ordenadorpresentan inconvenientes cuando se utilizan de manera aislada. La solución aconsejable es el empleo de modelos numéricos no lineales, contrastados y validados con evidencia experimental. Ésta es la práctica que se ha seguido en los últimos años a nivel internacional, mediante la colaboración de diversos organismos representativos de la industria y de las autoridades reguladoras en EE.UU. y otros países.

Debido a la magnitud y el nivel de detalles existentes en una contención nuclear, se recomienda abordar el análisis mediante modelos numéricos en etapas diferenciadas, partiendo de modelos globales y concluyendo con modelos locales de detalles estructurales. Los modelos globales (generalmente axisimétricos o con simetría cíclica) proporcionan una primera información valiosa acerca de la respuesta estructural de la Contención, identificando los modos de colapso global. Los modelos locales se justifican por la posible existencia de modos de fallo local antes de alcanzar el fallo global, debido a los efectos de concentración de tensión y deformación que originan las discontinuida. des en la lámina como penetraciones o compuertas. Los modelos locales emplean generalmente mallas tridimensionales más detalladas, utilizando las condiciones de contorno obtenidas previamente del análisis del modelo global.

La modelización realista de contenciones de hormigón armado frente a los estados últimos provenientes de sobrepresiones internas presenta algunos problemas, debido a la dificultad de representar adecuadamente el comportamiento del material sometido a estados multiaxiles de tracción. Para estos estados el hormigón exhibe un comportamiento inestable. Es difícil encontrar un modelo suficientemente robusto y contras. tado que interprete correctamente tanto la etapa de aparición y desarrollo de las fisuras como el estado último de la contención, sometida a estados de tracción multiaxil que aparecen en sobrepresiones internas. A pesar de esto se han obtenido resultados aceptables en los casos analizados, empleando un programa de elementos finitos no lineal disponible en el mercado (ABAQUS). Asimismo se ha observado que existe un margen amplio de seguridad frente a los requisitos de diseño, desencadenándose el fallo a partir de sobrepresiones internas unas 3 veces superiores a las de diseño de la contención.
La modelización de contenciones metálicas ofrece menos problemas, siempre que se emplee un código no lineal suficientemente fiable y robusto. El acero presenta un comportamiento considerablemente más dúctil que el hormigón y por lo tanto presenta menos problemas numéricos por las inestabilidades. En la contención evaluada se constató asimismo un margen elevado de seguridad, del orden de 2,5 frente a la presión de diseño.

\section{AGRADECIMIENTOS}

El trabajo descrito en este artículo ha sido realizado en sus diversas fases con financiación del Centro Común de Investigación de las Comunidades Europeas (Ispra), Consejo de Seguridad Nuclear, UNESA y NUCLENOR. Se agradece el apoyo prestado por los citados organismos. Agradecemos asimismo la ayuda prestada por D. Joaquín Martí, D. Francisco Beltrán y D. Gonzalo González de PRINCIPIA S.A., coautores de los trabajos reseñados.

\section{REFERENCIAS}

1. Zienkiewicz, O.C.: El método de los Elementos Finitos. Ed. Reverté, 1980.

2. Hughes, T.J.R.: The Finite Element Method. Linear Static and Dynamic Finite Element Analysis. Prentice-Hall, 1987.

3. Courant, R.: Variational Methods for the Solution of Problems of Equilibrium and Vibrations. Bull. Amer. Math. Soc. 49, pp. $1-23$ (1943).

4. Argyris, J.H. y Kelsey, S.: Energy Theorems and Structural Analysis. Aircraft Eng., Oct. 1984.

5. Clough, R.W.: The Finite Element in Plane Stress Analysis. 2nd. ASCE Conf. on Electronic Computation, Pittsburg PA, 1960.

6. Hibbit, H.D., Marcal, P.V. y Rice, J.R.: A Finite Element Formulation for Problems of Large Strains and Displacements. Int. J. Solids \& Struct., vol. 6, pp. 1069-1086, 1970.

7. Yaghmai, S. and Popov, E.P.: Incremental Analysis of Large Deflections of Shells of Revolution. Int. J. Solids \& Struct. Vol. 7, pp. 1375-1393, 1971.

8. Malvern, L.E.: Introduction to the Mechanics of a Continuous Medium. Prentice-Hall, 1969.

9. Krieg, R.D. y Key, S.W.: Implementation of a Timeindependent Plasticity Theory into Structural Computer Programs. en Constitutive Equations in Viscoplasticity, J.A. Stricklin y K.J. Saczalski eds., ASME winter mtg., Nueva York Dic. 1976. 
10. Hill, R.: The Mathematical Theory of Plasticity. Clarendon Press, Oxford 1950.

11. NEA Group of Experts.: Severe Accidents in Nuclear Power Plants. Nuclear Energy Agency. OECD, París 1986.

12. Belytschko, T.: An Overview of Semidiscretization and Time-Integration Methods. en Computational Methods for Transient Analysis, T. Belytschko y T.J.R. Hughes eds., North-Holland, Amsterdam (1983).

13. Prevost, J.H.: Plasticity Theory for Soil StressStrain behaviour. J. Eng. Mech. (ASCE) vol. 104, pp. 1177-1194, 1978.

14. Schofield, A.H. y Wroth, C.P.: Critical State Soil Mechanics. McGraw-Hill, 1968.

15. Willam, K.J. y Warnke, E.P.: Constitutive Model for the Triaxial Behaviour of Concrete. Proc. Int. Assoc. of Bridge and Struct. Engr., Seminar on Concrete Struct. Subjected to Triaxial Stresses, Paper III-1, Bergamo, Italia 1974.

16. Ottosen, N.S.: A failure Criterion for Concrete. J. Engrg. Mech. ASCE 103 (EM4), pp. 527-535 (1977).

17. Chen, A.C.T. y Chen, W.F.: Constitutive Relations for Concrete. J. Eng. Mech. Div. ASCE vol. 101, EM4, pp. 461-481 (1975).

18. Bazant, Z.P.: Mechanics of Distributed Cracking. Appl. mech. Rev. vol. 39 no. 5 (1985).

19. Nagtegaal, J.C., Parks, D.M. y Rice, J.R.: On Numerically Accurate Finite Element Solutions in the Fully Plastic range. Comp. Meth. Appl. Mech. \& Eng., vol. 4, pp. 153-177 (1974).

20. Oñate, E., Suárez, B. y Zienkiewicz, O.C.: Formulación de nuevos Elementos para Análisis de Placas Delgadas y Gruesas. Memorias I congreso de Métodos Numéricos en Ingeniería, eds. G. Winter y M. Galante, pp. 409-422 (1990).

21. Bathe, K.J. y Dvorkin, E.N.: A Four Node Plate Bending Element Based on Mindlin/Reissner Plate Theory and Mixed Interpolation. Int. J. Numer. Meth. Eng., vol. 21 pp. $367-383$ (1985)

22. Felippa, C.A. y Militello, C.: The ANDES formulation of Finite Elements. Memorias I congreso de Métodos Numéricos en Ingeniería, eds. G. Winter y M. Galante, pp. 30-41 (1990).

23. Matthies, H. y Strang, G.: The Solution of Nonlinear Finite Element Equations. Int. J. Numer. Meth. Eng., vol. 14 pp. 1613-1626 (1979).
24. Underwood, P.: Dynamic Relaxation. en Computational Methods for Transient Analysis, T. Belytschko y T.J.R. Hughes eds., North-Holland, Amsterdam (1983).

25. Crisfield, M.A.: A Fast Incremental/Iterative Solution Procedure that Handles Snap Through. Computers and Structures, vol. 133 pp. 55-62 (1981).

26. Bathe, K.J.: ADINA - Automatic Dynamic Incrementation for Nonlinear Analysis. Documentación del programa.

27. Teague, H.J. The Role of Reactor Containment in Controlling Severe Accidents. NEA Newsletter. Spring 1989.

28. Parks, M.B.; Horschel, D.S; Riesemann, W.A von. Summary of NRC-Sponsored Research on Containment Integrity. 2nd International Conference on Containment Design and Operation. Toronto, Octubre 14-17 1990.

29. Davis, G.A; Matzie, R.A; Green, M.D; Brewer, H.D; Fox, W.A. Design Considerations for Severe Accident Containment Performance. 4th Workshop on Containment Integrity. Arlington, Virginia 1988.

30. CBI Na-Con, Inc. Mark I Containment Severe Accident Analysis. Report prepared for the Mark I Owners Group, 1987.

31. Principia, S.A. Seminario sobre Comportamiento Estructural de la Contención. Proyecto ACEEspaña, Abril 1990.

32. Technica/Principia/ETSICCP. Analytical Capability for Predicting Structural Response of NPP Concrete Containments to Severe Loads. Final Report to Comission of the European Communities. Joint Research Centre, Ispra 1989.

33. Principia, S.A. Análisis del Experimento de Presurización Interna de la Contención de Sandia a Escala 1:6. Informe para Unidad Eléctrica, S.A. Noviembre 1989.

34. Clauss, D.B. Round Robin Pretest Analyses of a 1:6 Scale Reinforced Concrete Containment Model Subject to Static Internal Pressurization. NUREG/CR-4913; SAN87-4913, 1987.

35. Hibbitt, Karlsson \& Sorensen, Inc. ABAQUS, User's Manual. HKS Inc; Providence 1987. 